\title{
98. On Factor Set of the Third Obstruction
}

\author{
By Katuhiko Mizuno \\ Department of Mathematics, Osaka City University \\ (Comm. by K. Kunugi, M.J.A., July 12, 1955)
}

The object of the present note ${ }^{1)}$ is to give the third obstruction theorem for mappings of a geometric complex $K$ into a topological space $Y$ such that

$$
\pi_{i}(Y)=0 \text { for } 0 \leq i<n, n<i<q \text {, and } q<i<r<2 q-1 \text {, }
$$

along the line of Eilenberg-MacLane. ${ }^{2)}$

For such a space $Y$ we described previously ${ }^{3)}$ the cohomology class $\boldsymbol{k}_{n . q}^{r+1}$ of $H^{r+1}\left(\pi_{n}, \pi_{q}, \boldsymbol{k}_{n}^{q+1} ; \pi_{r}\right)^{4)}$ as a topological invariant if we pay no heed to the identification of the complexes $K\left(\pi_{n}, n, \pi_{q}, q, k\right)$, where $\boldsymbol{k}_{n}^{q+1}=\boldsymbol{k}_{n}^{q+1}(Y)$ is the Eilenberg-MacLane invariant of the space $Y$.

In this paper we shall introduce new operators $\boldsymbol{y}_{\mathrm{r}}$ and $\boldsymbol{y}_{\tau}$. And by making use of $\boldsymbol{k}_{n . q}^{r+1}, \boldsymbol{k}_{n . q}^{r+1}$, we shall describe a factor set of the third obstruction of a map.

1. As a preliminary to the definition of the basic operators, we shall consider first certain maps.

We wish to classify simplicial maps $T$ of a C.S.S. complex $K$ in $K\left(\Pi, n, \Pi^{\prime}, q, k\right)$. Such a map determines a cocycle $x_{n}=T^{\sharp} b_{n} \in$ $Z^{n}(K ; \Pi)$ and a cochain $x_{q}=T^{\#} b_{q} \in C^{q}\left(K ; \Pi^{\prime}\right)$, where $b_{n}$ is the basic cocycle in $Z^{n}\left(\Pi, n, \Pi^{\prime}, q, k ; \Pi\right) \cong Z^{n}(\Pi, n ; \Pi)$ and $b_{q}$ is the basic cochain in $C^{q}\left(\Pi, n, \Pi^{\prime}, q, k ; \Pi^{\prime}\right)$ defined by setting

$$
b_{n}(\phi, \psi)=\phi\left(\varepsilon_{n}\right), \quad b_{q}(\phi, \psi)=\psi\left(\varepsilon_{q}\right) .
$$

Lemma 1. Given the complex $K\left(\Pi, n, \Pi^{\prime}, q, k\right)$ and the C.S.S. complex $K$, the rule $T \rightarrow\left(x_{n}, x_{q}\right)$ establishes a one to one correspondence between simplicial maps and pairs $\left(x_{n}, x_{q}\right)$ satisfying the conditions:

$$
x_{n} \in Z^{n}(K ; \Pi), x_{q} \in C^{q}\left(K ; \Pi^{\prime}\right), k T\left(x_{n}\right)+\delta x_{q}=0 .
$$

The map $T$ corresponding in this fashion to the pair $\left(x_{n}, x_{q}\right)$ will be denoted by $T\left(x_{n}, x_{q}\right)$. Then $T\left(x_{n}, x_{q}\right)$ is characterized as

1) Full details will appear in the Journal of the Institute of Polytechnics, Osaka City University.

2) S. Eilenberg and S. MacLane: On the groups $H(\Pi, n)$, III, Ann. Math., 60, 513-557 (1954). Present note makes full use of the results and terminology of this paper.

3) K. Mizuno: On the minimal complexes, Jour. Inst. Polytech., Osaka City Univ., 5, 41-51 (1954).

4) For the sake of brevity, we write in the following $\pi_{n}=\pi_{n}(Y), \pi_{q}=\pi_{q}(Y)$, and $\pi_{r}=\pi_{r}(Y)$. 


$$
T\left(x_{n}, x_{q}\right)=\gamma\left[i_{n} \times i_{q}\right]\left[T\left(x_{n}\right) \times T\left(x_{q}\right)\right] e
$$

where $i_{n}$ and $i_{q}$ are natural inclusions.

For our future convenience, we now derive an explicit formula for the automorphism $\eta(\phi, \psi)=\left(\phi^{\prime}, \psi^{\prime}\right)$ such that

$$
\begin{aligned}
& \eta: K\left(\Pi, n, \Pi^{\prime}, q, k\right) \rightarrow K\left(\Pi, n, \Pi^{\prime}, q, k\right) \\
& \phi \equiv \phi^{\prime} \quad \text { for any }(\phi, \psi) \text { of } K\left(\Pi, n, \Pi^{\prime}, q, k\right) .
\end{aligned}
$$

According to Lemma 1 , such a map $\eta$ is represented as $T\left(b_{n}, b_{q}^{\prime}\right)$ where $b_{n}$ is the basic cocycle defined above and $b_{q}^{\prime}=\eta^{\#} b_{q}$ is a cochain of $C^{q}\left(\Pi, n, \Pi^{\prime}, q, k ; \Pi^{\prime}\right)$. Generally $b_{q}^{\prime}$ is different from $b_{q}$ and induces a cocycle $h_{q}=b_{q}^{\prime}-b_{q}$ of $Z^{q}\left(\Pi, n, \Pi^{\prime}, q, k ; \Pi^{\prime}\right)$.

Lemma 2. Given the complex $K\left(\Pi, n, \Pi^{\prime}, q, k\right)$, the rule $\eta \rightarrow h_{q}$ establishes a one to one correspondence between the chain homotopic class of $\eta$ and the cohomology class of $h_{q}$.

The map $\eta$ corresponding in this fashion to the cocycle $h_{q}$ will be denoted by $\eta\left(h_{q}\right)$, then $\eta\left(h_{q}\right)$ is characterized as

$$
\eta\left(h_{q}\right)=T\left(b_{n}, b_{q}\right) \circ i_{q} T\left(h_{q}\right)
$$

where $\circ$ is the internal product in the complex $K\left(\Pi, n, \Pi^{\prime}, q, k\right)$ and $T\left(b_{n}, b_{q}\right)$ is obviously the identity map.

If we replace $x_{q}$ in the formula $T\left(x_{n}, x_{q}\right)$ by another $x_{q}^{\prime}$, we have a cocycle $d_{q}=x_{q}^{\prime}-x_{q} \in Z^{q}\left(K ; \Pi^{\prime}\right)$, and successively, the map $T\left(x_{n}, x_{q}^{\prime}\right)$ is represented by

$$
T\left(x_{n}, x_{q}^{\prime}\right)=T\left(x_{n}, x_{q}\right) \circ i_{q} T\left(d_{q}\right) .
$$

Therefore if we identify the complex $K\left(\Pi, n, \Pi^{\prime}, q, k\right)$ with the image of the automorphism $\eta$, we can identify $T\left(x_{n}, x_{q}^{\prime}\right)$ with $T\left(x_{n}, x_{q}\right)$. Then we shall define $\tau\left(x_{n}\right)$ as the family of $T\left(x_{n}, x_{q}\right)$ where $x_{n}$ is a fixed cocycle of $Z^{n}(K ; \Pi)$ satisfying $k T\left(x_{n}\right) \sim 0$.

Lemma 3. The cocycles $x_{n}^{1}, x_{n}^{2} \in Z^{n}(K ; \Pi)$ such that $k T\left(x_{n}^{1}\right) \sim 0$ $\sim k T\left(x_{n}^{2}\right)$ are cohomologous if and only if the families $\tau\left(x_{n}^{1}\right), \tau\left(x_{n}^{2}\right)$ are chain homotopic. ${ }^{5)}$

Given two C.S.S. pairs $\left(K, L_{1}\right),\left(K, L_{2}\right)$ and two cocycles $x_{n} \epsilon$ $Z^{n}\left(K, L_{1} ; \Pi\right), x_{q} \in Z^{q}\left(K, L_{2} ; \Pi^{\prime}\right)$, we shall define a chain transformation

$$
\gamma_{n . q}\left(x_{n}, x_{q}\right):(K, L) \rightarrow K\left(\Pi, n, \Pi^{\prime}, q, k\right)
$$

for each simplex $\sigma(\operatorname{dim} \sigma \leq 2 q)$ as

$$
\gamma_{n . q}\left(x_{n}, x_{q}\right) \sigma=\gamma g\left[i_{n} \otimes i_{q}\right]\left[R\left(x_{n}\right) \otimes R\left(x_{q}\right)\right] f e \sigma
$$

where $L$ is the union of the subcomplexes $L_{1}, L_{2}$.

Replacement of $x_{n}$ or $x_{q}$ by a cohomologous cocycle replaces $R\left(x_{n}\right)$ or $R\left(x_{q}\right)$ by a chain homotopic map, therefore, the homotopy class of the map $\gamma_{n . q}$ depends only on the cohomology classes $\boldsymbol{x}_{n}, \boldsymbol{x}_{q}$ of $x_{n}, x_{q}$ respectively.

2. Take abelian groups $\Pi, \Pi^{\prime}$, and $G$, positive integers $n, q$, and

5) Namely, $\tau\left(x_{n}^{1}\right)$ and $\tau\left(x_{n}^{2}\right)$ contain $T\left(x_{n}^{1}, x_{q}^{1}\right), T\left(x_{n}^{2}, x_{q}^{2}\right): K \rightarrow K\left(\Pi, n, \Pi^{\prime}, q, k\right)$ respectively and $T\left(x_{n}^{1}, x_{q}^{1}\right) \cong T\left(x_{n}^{2}, x_{q}^{2}\right)$. 
$r(1<n<q<r<2 q)$, a cohomology class $k \in H^{q+1}\left(\Pi, n ; \Pi^{\prime}\right)$, and a cohomology class $\boldsymbol{y} \in H^{r}\left(\Pi, n, \Pi^{\prime}, q, k ; G\right)$ where $k$ is a fixed cocycle of $\boldsymbol{y}$.

The $\gamma$-operator $\boldsymbol{y}_{\gamma}$ is defined for cohomology classes $\boldsymbol{x}_{n} \epsilon$ $H^{n}\left(K, L_{1} ; \Pi\right)$ and $\boldsymbol{x}_{q} \in H^{q}\left(K, L_{2} ; \Pi^{\prime}\right)$ by the formula

$$
\boldsymbol{y}_{\Upsilon}\left(\boldsymbol{x}_{n}, \boldsymbol{x}_{q}\right)=\gamma_{n . q}\left(\boldsymbol{x}_{n}, \boldsymbol{x}_{q}\right)^{*} \boldsymbol{y}
$$

this is an element of $H^{r}(K, L ; G)$.

Let $\boldsymbol{x}_{n} \in H^{n}(K ; \Pi)$ be a cohomology class whose representative cocycle $x_{n}$ satisfies $k T\left(x_{n}\right) \sim 0$. The $\tau$-operator $\boldsymbol{y}_{\tau}$ is defined for such a class $\boldsymbol{x}_{n}$ by the formula

$$
\boldsymbol{y}_{\tau}\left(\boldsymbol{x}_{n}\right)=\tau\left(\boldsymbol{x}_{n}\right)^{*} \boldsymbol{y}
$$

this is a family of elements of $H^{r}(K ; G)$.

Theorem 4. For $\boldsymbol{x}_{n} \in H^{n}(K ; \Pi)$ such that $\boldsymbol{k} \vdash \boldsymbol{x}_{n}=0$, we can determine an element $\boldsymbol{y}_{\tau}\left(\boldsymbol{x}_{n}\right)$ of the factor group $H^{r}(K ; G) \bmod$ $\boldsymbol{y}_{\mathrm{r}}\left(\boldsymbol{x}_{n}, H^{q}\left(K ; \Pi^{\prime}\right)\right)+i_{n}^{*} \boldsymbol{y} \vdash H^{q}\left(K ; \Pi^{\prime}\right)$ whose generator is represented by the formula $\boldsymbol{y}_{r}\left(\boldsymbol{x}_{n}, \boldsymbol{x}_{q}\right)+i_{q}^{*} \boldsymbol{y} \vdash \boldsymbol{x}_{q}$, where $\boldsymbol{x}_{q}$ is any cohomology class going round the group $H^{q}\left(K ; \Pi^{\prime}\right)$.

3. It is well known that the cohomology classes $\boldsymbol{k}_{n}^{q+1}(Y) \epsilon$ $H^{q+1}\left(\pi_{n}, n ; \pi_{q}\right)$ and $\boldsymbol{k}_{q}^{r+1}(Y) \in H^{r+1}\left(\pi_{q}, q ; \pi_{r}\right)$ attach to the space $Y$ as topological invariants. And, it is obvious from our definition that $i_{q}^{*} \boldsymbol{k}_{n \cdot q}^{r+1}=\boldsymbol{k}_{q}^{r+1}$.

In the identification of the complexes $K\left(\pi_{n}, n, \pi_{q}, q, k\right)$, the only essential part is the identification of the complex with the image of the automorphism $\eta$ about which we considered above. Namely, we can recognize the invariant as the family $\left\{\eta\left(\boldsymbol{h}_{q}\right)^{*} \boldsymbol{k}_{n . q}^{r+1}\right\}$ of the classes of $H^{r+1}\left(\pi_{n}, n, \pi_{q}, q, k ; \pi_{r}\right)$ for the fixed complex $K\left(\pi_{n}, n, \pi_{q}\right.$, $q, k)$, where $\boldsymbol{h}_{q}$ is the cohomology class going round the $H^{q}\left(\pi_{n}, n, \pi_{q}\right.$, $\left.q, k ; \pi_{q}\right) \cong H^{q}\left(Y ; \pi_{q}\right)$. In other words, the invariant is the image $\tau\left(\boldsymbol{b}_{n}\right)^{*} \boldsymbol{k}_{n \cdot q}^{r+1}$ of $\boldsymbol{k}_{n . q}^{r+1}$, and is an element of the factor group $H^{r+1}\left(\pi_{n}, \pi_{q}\right.$, $\left.\boldsymbol{k}_{n}^{\prime+1} ; \pi_{r}\right) \bmod \boldsymbol{k}_{n \cdot q}^{r+1}\left(\boldsymbol{b}_{n}, H^{q}\left(\pi_{n}, \pi_{q}, \boldsymbol{k}_{n}^{q+1} ; \pi_{q}\right)\right)+\boldsymbol{k}_{q}^{r+1} \vdash H^{q}\left(\pi_{n}, \pi_{q}, \boldsymbol{k}_{n}^{\prime+1} ; \pi_{q}\right)$. In the following we shall denote this element simply as $\left\{\boldsymbol{k}_{n, q}^{r+1}\right\}$.

4. Let $f: K^{n} L \rightarrow Y$ be a map extendible to a map $K^{n+1} \smile L \rightarrow Y$ with $f\left(K^{n-1}\right)=y_{0}$ which is the base point of $Y$, then a characteristic cocycle $a^{n}(f) \in Z^{n}\left(K ; \pi_{n}\right)$ is determined as usual. If the second obstruction $z^{q+1}(f)=0$, the map $f$ admits an extension $f^{\prime}: K^{r} \cup L \rightarrow Y$ and has an obstruction $c^{r+1}\left(f^{\prime}\right) \in Z^{r+1}\left(K, L ; \pi_{r}\right)$. The cohomology class $\boldsymbol{z}^{r+1}\left(f^{\prime}\right)$ of this cocycle depends on the choice of the extension $f^{\prime} \mid K^{q} \smile L$ as follows.

It follows from $z^{q+1}(f)=0$ that there is a cochain $a^{q}\left(f^{\prime}\right)$ in $C^{q}\left(K ; \pi_{q}\right)$ satisfying $k T\left(a^{n}(f)\right)+\delta a^{q}\left(f^{\prime}\right)=0$.

Theorem 5. Let $f_{1}, f_{2}: K^{q} \sqcup L \rightarrow Y$ be two extensions of the map $f$ above and which are extendible to $K^{q+1} \cup L \rightarrow Y$. Then

$$
\boldsymbol{z}^{r+1}\left(f_{1}\right)-\boldsymbol{z}^{r+1}\left(f_{2}\right)=\boldsymbol{k}_{n . q}^{r+1} r\left(\boldsymbol{a}^{n}(f), \boldsymbol{a}^{q}\left(f_{1}, f_{2}\right)\right)+\boldsymbol{k}_{q}^{r+1} \vdash \boldsymbol{a}^{q}\left(f_{1}, f_{2}\right),
$$


where $\boldsymbol{a}^{q}\left(f_{1}, f_{2}\right) \in H^{q}\left(K, L ; \pi_{q}\right)$ is the cohomology class of the cocycle $a^{q}\left(f_{1}\right)-a^{q}\left(f_{2}\right)$, and $\boldsymbol{a}^{n}(f) \in H^{q}\left(K ; \pi_{n}\right)$ is the cohomology class of the cocycle $a^{n}(f)$.

Theorem 6. Let $f: K^{n} \rightarrow Y$ be a map extendible to a map $K^{a+1} \rightarrow Y$, then the third obstruction of $f$ is determined as follows:

$$
\left\{\boldsymbol{z}^{r+1}(f)\right\}=\boldsymbol{k}_{n \cdot \% \tau}^{r+1} \boldsymbol{a}^{n}(f) \text {. }
$$

5. We shall display a few properties of $\gamma$-and $\tau$-operators in some special cases in the following.

a) If $n+q>r$, then $\boldsymbol{y}_{r}\left(\boldsymbol{x}_{n}, \boldsymbol{x}_{q}\right)=0$.

b) If $n+q=r$, then $\boldsymbol{y}_{r}\left(\boldsymbol{x}_{n}, \boldsymbol{x}_{q}\right)=\boldsymbol{x}_{n} \smile \boldsymbol{x}_{q}$ where the cup product is taken relative to the pairing determined by $\boldsymbol{y}$. Especially if $\boldsymbol{y}$ is a representative class of the invariant $\left\{\boldsymbol{k}_{n \cdot q}^{r+1}\right\}$, the cup product are paired by the Whitehead product.

c) If $n>2, r=q+1$ then the invariant $\left\{\boldsymbol{k}_{n . q}^{r+1}\right\}$ is determined as a coset of $H^{r+1}\left(\pi_{n}, \pi_{q}, \boldsymbol{k}_{n}^{i+1} ; \pi_{r}\right) \bmod S q^{2} H^{q}\left(\pi_{n}, \pi_{q}, \boldsymbol{k}_{n}^{q+1} ; \pi_{q}\right)$. And the third obstruction is also determined as a coset of $H^{r+1}\left(K ; \pi_{r}\right) \bmod$ $S q^{2} H^{q}\left(K ; \pi_{q}\right){ }^{6)}$

d) If $n=2, q=3, r=4$ then the third obstruction of a map $f: K^{2} \rightarrow Y$ is determined as a coset of $H^{5}\left(K ; \pi_{4}\right) \bmod \boldsymbol{a}^{2}(f) \smile H^{2}$ $\left(K ; \pi_{3}\right)+S q^{2} H^{3}\left(K ; \pi_{3}\right)^{6}{ }^{6}$

6) Refer. N. Shimada and H. Uehara: On a homotopy classification of mappings of an $(n+1)$ dimensional complex into an arcwise connected topological space which is aspherical in dimensions less than $n(n>2)$, Nagoya Math. Jour., 3, 67-72 (1951). 